\title{
Effects of the sleep quality of chronic stroke outpatients on patterns of activity performance and quality of life
}

\author{
JuHyung Park ${ }^{*}$
}

${ }^{1}$ Department of Occupational Therapy, Health Science College, Cheongju University, Republic of Korea

\begin{abstract}
This study examined the effects of the sleep quality of chronic stroke outpatients on their patterns of activity performance and quality of life. This study was conducted on 49 patients with chronic stroke who received outpatient care in hospitals in South Korea.The Pittsburgh Sleep Quality Index (PSQI) was used to examine sleep quality, the Occupational Questionnaire (OQ) was used to assess frequency of occupational participation; while the World Health Organization Quality of Life-BREF (WHOQOL-BREF)was used to measure quality of life. As the results of the analysis, it was shown that the patient group with higher quality of sleep in the surveyed chronic stroke outpatients were indicated to have the higher activity frequency in the work and daily living work in terms of their activity execution phase, whereas the patient group with higher quality of sleep the higher activity frequency in the field of the rest $(p<0.05)$. In addition, the higher quality of sleep group showed statistically significant higher quality of life $(p<0.05)$. The findings of this study showed that the sleep quality of outpatients with chronic stroke is related with their pattern of activity performance and quality of life, and that bad sleep quality may negatively affect their pattern of activity performance and quality of life.
\end{abstract}

Keywords: Quality of life, quality of sleep, stroke

\section{INTRODUCTION}

Due to their sudden disease and side effects, patients with chronic stroke encounter a variety of psycho-social problems, as they experience difficulties in performing the tasks they had performed independently before the onset of stroke (Hadidi, Huna Wagner, \& Lindquist, 2017). Once capable of healthy daily living, the individual experiences physical disabilities such as impaired comprehension, communication disabilities, and sensory

*Correspondence: juhyungi79@ahanmail.net

JuHyung Park, 298 Daesung-ro, Cheongwon-gu, Cheongju 363-764,

Republic of Korea

Phone: +82-10-7751-7238 Fax: +82-43-229-7980

Received: 30 August 2018 Accepted: 31 October 2018

Sleep and Hypnosis

Journal homepage:

www.sleepandhypnosis.org

ISSN: 2458-9101 (Online) and motor skill loss, resulting in stress that mostly leads through a variety of psychological changes into insecurity, depression, embitterment disorder, emotional incontinence, etc (Kim et al., 2000). Previous studies have identified many factors of these psychological disorders, which mostly include depression, despair, stress, drug, social support, and familial relationships (Kim et al., 2007). These psychological factors interrupt the subject's sleep, which is essential for rest and recharging during daily living, resulting in sleeping disorders, which have been reported as a frequent problem for patients with neurological diseases, including stroke (Leppavuori, Pohjasvaara, Vataja, Kaste, \& Erkinjuntti, 2002; Palomaki et al., 2003).

As a basic human need, good sleep provides rest and stabilization as an essential for physiological and psychological homeostasis (Hu \& Silberfarb, 1991). However, it has been reported that about $20 \sim 50 \%$ of 
patients with chronic stroke have sleep disturbance, showing typical symptoms such as insomnia, lethargy, diurnal rhythm sleep disturbance, sleep apnea, fatigue, etc (Verma, Anand, \& Verma, 2007). Previous research findings suggest that sleep disturbance affects cognitive deficiency, as well as reduction in attention, spatial capacities, memory, time orientation, and psycho-social functions, to negatively affect daily activity performance and quality of life (Siengsukon \& Boyd, 2008). These findings are also applicable to chronic stroke patients.

If we review recent studies on the quality of sleep of stroke patients, in particular, the studies that examined the relationship between sleep quality and functional level in 80 acute stroke patients, they reported that there was a significant correlation between sleep quality, upper extremity function, and ability to perform daily activities, and it is important to manage the factors that interfere with their sleep in terms of their ability to perform daily activities and return to society (Kim, Kim, Yang, Kim \& Kim, 2015). Furthermore, in the studies on the relationship between quality of sleep, stress level and rehabilitation motivation of patients with chronic stroke for more than 6 months after the onset of stroke, it was reported that their sleep quality has a significantly correlation with stress and rehabilitation motivation, which are critical factors in the rehabilitation of the subjects (Kang, Hong \& Jung, 2017). However, only a few studies have been conducted on the sleep quality of stroke patients, which means there are even fewer practical studies on sleep quality level and its effects on chronic stroke outpatients, who are exposed and required to perform various roles and activities in their local communities without certain control or management of their lives after the initial hospitalization stage. For this reason, this study aims to examine the affection of sleep quality on their daily activity patterns and quality of life in outpatients with chronic stroke.

\section{METHOD}

This research was conducted on 49 patients who received outpatient care in hospitals in South Korea after being diagnosed with hemiplegia by stroke. The subject selection criteria was a score of 24 or higher on the
Table 1. General characteristic of the subjects

\begin{tabular}{lll}
\hline & Mean \pm SD & Frequency (\%) \\
\hline Age(year) & $69.98 \pm 10.83$ & \\
Onset(month) & $22.57 \pm 11.67$ & \\
$\quad$ Gender & & \\
$\quad$ Male & & $29(59.18 \%)$ \\
Female & & $20(40.81 \%)$ \\
Stroke type & \\
$\quad$ Infarction & $41((83.67 \%)$ \\
$\quad$ Hemorrhage & & $8(16.3 \%)$ \\
Paretic side(Right/Left) & & \\
$\quad$ Right & & $24(48.97 \%)$ \\
$\quad$ Left & $25(51.02 \%)$ \\
\hline
\end{tabular}

Korean version of Mini-Mental State Examination (MMSE-K), as the cognitive level for the reliability of the survey results, those who have passed 6 months or longer after the onset of stroke, and those with no disease other than stroke and no aphasia. And, those who are taking medications in relation to sleep are also excluded from the list of subjects. All subjects were fully informed of the purpose of this study before submitting their voluntary consent to participate in the study. This study complied with the ethical standards of the Declaration of Helsinki. Table 1 shows the general characteristics of the subjects. The sample size calculation was based on effect size 0.5 , $80 \%$ power, level of significance 0.05 , using G-power version 3.01. The required sample size was 29 .

The Pittsburgh Sleep Quality Index (PSQI) was used to measure the sleep quality of the subjects. A self-reporting tool, the PSQI assesses seven components - namely, sleep latency, sleep duration, use of sleeping medication, habitual sleep efficiency, subject sleep quality, sleep disturbance, and daytime dysfunction - using nineteen questions. Each component is measured on a scale of $0 \sim 3$; and a score of 5 or above indicates bad sleep quality. The reliability was Cronbach's $a=.83$ and the test-retest reliability was .85 (Tang, Grace Laru, Mok, Ungvari \& Wong, 2015; Zhang et al., 2014). The Occupational Questionnaire (OQ) was used to assess the subjects' pattern of activity performance as an assessment tool developed to check the subject's lifestyle through a timetable format designed to assess the daily pattern of activity performance (Smith, Kielhofner, \& Watts, 1986). The OQ surveys the subject's pattern of daily activity performance in four areas - performance domain, 
performance level, importance, and satisfaction - by recording each activity every 30 minutes from five in the morning to noon on a scale of 5 . The test-retest reliability was $68.0 \%$ for the activities by time-slot, $87.0 \%$ for the activity type, $77.0 \%$ for activity performance, $81.0 \%$ for the activity values, and $77.0 \%$ for the activity enjoyment level. The concurrent validity was compared with the Household Work Study Daily test, and showed a consistency of $82 \%$ for the activity type and of $90.0 \%$ for the individual activity classification method (Hong, Jang, $\&$ Seo, 2011). To examine the patterns of daily activity performance of the subjects, this study used the responses to the frequency of activity performance areas by time slot for the analysis. The World Heath Organization Quality of Life-BREF (WHOQOL-BREF) was used to measure the quality of life; the WHOQOL-BREF used the Korean version developed by Min, Lee and Kim of the convenient WHO quality of life scale, which is an assessment tool consisting of 26 questions, including 24 questions in four domains (physical health, psychology, social relationships, and environment) and two questions on overall quality of life. A higher score indicated higher quality of life. The reliability of the testing tool is Cronbach's alpha 0.89 (Min et al., 2002).

SPSS Version 18.0 was used for the statistical analysis in this study. Descriptive statistics and frequency analysis were applied to the general characteristics. The independent samples t-test was conducted on the group with higher sleep quality and another with lower sleep quality based on the PSQI score of 5 to examine the effect of the sleep quality on the subjects' pattern of activity performance and quality of life.

\section{RESULTS}

Table 2 shows the analytical findings on the differences in the pattern of activity performance and quality of life depending on sleep quality. If we investigate the results of the comparison of the frequency of performance by the individual areas according to the quality of sleep, it was shown that the group of relatively higher level of sleep quality, which was evaluated based on the PSQI 5 point score, had a statistically significant participation
Table 2. The Difference of $O Q$ and WHOQOL-BREF score to PSQI level

\begin{tabular}{lcc}
\hline & \multicolumn{2}{c}{ PSQI } \\
\cline { 2 - 3 } & $\begin{array}{c}\text { Good sleep quality } \\
\mathbf{N}=\mathbf{2 7 / M} \mathbf{M S D}\end{array}$ & $\begin{array}{c}\text { Bad sleep quality } \\
\mathbf{N}=\mathbf{2 2} / \mathbf{M} \pm \text { SD }\end{array}$ \\
\hline OQ & $11.20 \pm 6.36$ & $7.60 \pm 3.81$ \\
work* $^{*}$ & $8.80 \pm 5.33$ & $6.60 \pm 2.21$ \\
daily living work* $_{\text {recreation }}$ & $5.70 \pm 2.36$ & $5.40 \pm 4.37$ \\
rest* & $12.30 \pm 3.15$ & $18.40 \pm 5.60$ \\
WHOQOL-BREF* $^{*}$ & $81.74 \pm 6.87$ & $75.19 \pm 7.20$ \\
\hline
\end{tabular}

${ }^{*} p<0.05$. OQ: Occupational Questionnaire, QOS: Quality of Sleep, WHOQOL-BREF: World Heath Organization Quality of Life-BREF

frequency in the areas such as work and daily living work rather than that of lower level of sleep quality. However, the recreation area showed no significant difference in the frequency of participation, but on the contrary, in the rest area, the group with lower quality of sleep showed higher frequency of participation. As a result of survey in terms of quality of life, the group with high quality of sleep showed a statistically significant higher quality of life quality rather than the group with lower level of sleep quality (Table 2).

\section{DISCUSSION}

This study examined the effects of the sleep quality of chronic stroke outpatients on their pattern of activity performance and quality of life. The findings of this study showed that the sleep quality of chronic stroke outpatients significantly affects their daily pattern of activity performance and quality of life.

The aspect in which people perform activities during a day means their unique lifestyle. The activities performed during a day by each people give affection on the successful pursuit of meaningful activities for the modern people. However, if each of these daily performance activities is shifted to one side, the rest of the area will become relatively neglected, which will adversely affect human general health and quality of life (Bejerholm, 2010). Patterns of activity performance were compared by checking the frequency of each area using the OQ on the tasks and activities performed in a day. For the areas of work and daily living work in the group 
with higher sleep quality, the activity frequencies were statistically significantly higher than the group with lower sleep quality. The area of rest showed a statistically significantly lower activity frequency. These findings suggest that activities in the areas of work and daily living work, which are more proactive in a day, are performed more in the group with higher sleep quality. These results are in line with the previous research findings that reported that higher sleep quality results in improvements in the capacities of daily activities and rehabilitation motivation ( Ju, \& Kim, 2017; Kang, Hong, $\&$ Jung, 2017), supplementing and supporting the previous studies by analyzing the practical activity performance in a day. In addition, this means that when the quality of sleep of the subjects who are partially unbalanced in the life by the disability due to stroke is lower, the rate of active participation of the subjects for health could be lower in terms of daily life, and the percentage of passive activities of them could be increased. Also, the above findings showed significantly higher quality of life among the subjects in the group with higher sleep quality, which is in line with the previous research findings that mentioned significant correlations between the sleep quality and quality of life of stroke patients (Kim, Cha, Lee, \& Yoo, 2018). However, the subjects of this study are limited to chronic stroke patients who receive outpatient care, and thus presumably better reflect the characteristics of the sleep quality of chronic stroke patients who must be independent in their daily routines without separate management by the local community, in contrast to previous studies that have focused on all stroke patients, either hospitalized or receiving outpatient care (Kim, Cha, Lee, \& Yoo, 2018). The above findings suggest the importance of individual intervention and observation on sleep quality in rehabilitation to encourage chronic stroke patients to participate in their local communities, as sleep quality may affect healthy activity performance and quality of life. Limitations of this study include the fact that it only used subjective assessment tools on sleep quality, quality of life, and pattern of activity performance. Further studies should use more objective indicators for measurement and analysis.

Acknowledgements: None declared.

Funding: The authors declare that the current study was not financially supported by any institution or organization.

Informed Consent: Informed consent was obtained from all individual participants included in the study.

Conflict of Interest: The authors declare no conflict of interest.

\section{References}

Bejerholm, U. (2010). Occupational balance in people with schizophrenia. Occupational Therapy in Mental Health, 26(1), 1-17.

Hadidi, N. N., Huna Wagner, R. L., \& Lindquist, R. (2017). Nonpharmacological treatments for post-stroke depression: An integrative review of the literature. Research in Gerontological Nursing, 10(4), 182-195.

Hong, S. P., Jang, M. N., \& Seo, E. K. (2011). Time use among people with tetraplegia participating in wheelchair rugby. Journal of Korean Society of Occupational Therapy, 19(2), 1-12.

Hu, D. S., \& Silberfarb, P. M.. (1991). Management of sleep problems in cancer patients. Oncologyny, 5(9), 23-27.

Ju, S. H., \& Kim, H. (2017). Impact of sleep quality and pain degree on the activities of daily living in patients with stroke. Journal of Korean Society of Occupational Therapy, 25(3), 27-39.

Kang, M.S., Hong, K.H., \& Jung, H.R. (2017). The Relationship of Quality of Sleep With Stress and Rehabilitation Motivation in Stroke. Journal of Korean Society of Occupational Therapy, 25(3), 59-70.
Kim, H. O., Cha, T. H., Lee, J. S., \& Yoo, D. H. (2018). The effect of sleep quality and depressive symptom on quality of life for stroke patients. Journal of Korean Society of Occupational Therapy, 26(2), 43-53.

Kim, J., Kim, Y., Yang, K. I., Kim, D. E., \& Kim, S. A. (2015). The relationship between sleep disturbance and functional status in mild stroke patients. Annals of Rehabilitation Medicine, 39(4), 545-552.

Kim, S. J., Kim, Y. S., Yoo, S. W., Lee, M. H., Yu, K. H., Ma, H., \& Lee, B. C. (2000). Depressive and anxiety symptoms in the acute stage of stroke. Korean Journal of Stroke, 2(1), 40-47.

Kim, W. O., Kang, H. S., Wang, M. J., Kim, J. H., \& Choi, J. Y. (2007) Relationship among activity of daily living, depression, and Quality Of Life (QOL) in patients with stroke. Journal of EastWest Nursing Research, 13(2), 138-146.

Leppavuori, A., Pohjasvaara, T., Vataja, R., Kaste, M., \& Erkinjuntti, T. (2002). Insomnia in ischemic stroke patients. Cerebrovascular Diseases, 14(2), 90-97. 
Min, S. K., Kim, K. I., Lee, C. L., Jung, Y. C., Suh, S. Y., \& Kim, D. K. (2002). Development of the Korean versions of WHO Quality of Life scale and WHOQOL-BREF. Quality of Life Research, 11(6), 593-600.

Palomaki, H., Berg, A., Meririnne, E., Kaste, M., Lönnqvist, R., Lehtihalmes, M., \& Lönnqvist, J. (2003). Complaints of poststroke insomnia and its treatment with mianserin. Cerebrovascular Diseases, 15(1-2), 56-62.

Siengsukon, C. F., \& Boyd, L. A. (2008). Sleep enhances implicit motor skill learning in individuals poststroke. Topics in Stroke Rehabilitation, 15(1), 1-12.
Smith, N. R., Kielhofner, G., \& Watts, J. H. (1986). The relationship between volition, activity pattern and life satisfaction in the elderly. American Journal of Occupational Therapy, 40(4), 278-283.

Tang, W. K., Grace Laru, C., Mok, V., Ungvari, G. S., \& Wong, K. S. (2015). Insomnia and healthrelated quality of life in stroke. Topics in Stroke Rehabilitation, 22(3), 201-207.

Verma, A., Anand, V., \& Verma, N. P. (2007). Sleep disorders in chronic traumatic brain injury. Journal of Clinical Sleep Medicine, 3(4), 357-362.

Zhang, S., Chang, C., Zhang, J., Song, B., Fang, H., \& Xu, Y. (2014). Correlation analysis of sleep quality and youth ischemic stroke. Behavioural Neurology, 1-6. 